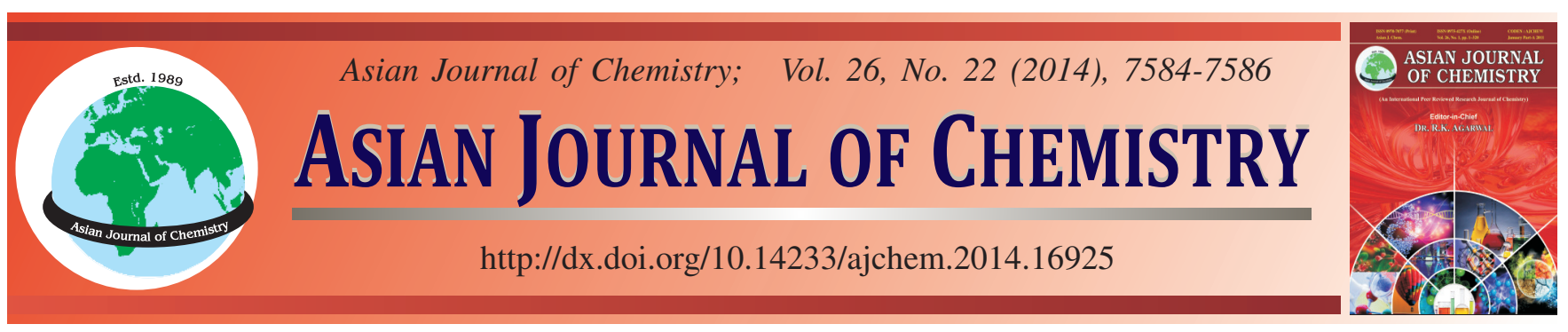

\title{
Preparation and Characterization of Porous Polyacrylonitrile Materials via Concentrated Emulsion Template Method
}

\author{
Haitao Chang ${ }^{*}$, Xueyong Gong, Chao Zheng and Zhenhua Wei
}

Institute of Chemical Engineering, Taishan Medical University, Tai'an 271000, P.R. China

*Corresponding author: Fax: +86 538 6229741; Tel: +86 538 6236207; E-mail: htchang@tsmc.edu.cn; changhaitao723@163.com

In this study, highly porous polyacrylonitrile materials have been prepared via concentrated emulsion template method. With the aid of colloidal silica nanoparticles added to the droplet phase stable concentrated emulsions had been obtained. To investigate their porous morphologies, the foams were characterized by scanning electron microscopy (SEM) and nitrogen adsorption. It was observed that silica nanoparticles acted as a cosurfactant, which was absorbed on the interface of the emulsion preventing the coalescence of the droplet phase. The cell diameters of polyacrylonitrile foams were controlled within a range of 1 to $10 \mu \mathrm{m}$ by varying the emulsion composition. Nitrogen adsorption analysis showed that the resulting foams possessed mesoporous pore structures. Our method provides a facile route to fabricate highly porous polyacrylonitrile materials with controlled morphology.

Keywords: Porous materials, Polymers, Polyacrylonitrile, Concentrated emulsion template.

ᄂ - - - - - - - - - - - - - - - - -

\section{INTRODUCTION}

Recently, highly porous polymer materials with controlled morphology prepared via concentrated emulsion template method had gained extensive research interests due to their favorable properties such as large surface area, high porosity, high absorbency capabilities and good permeability ${ }^{1,2}$. The concentrated emulsions had been defined as emulsions in which the droplet phase occupies more than $74 \%$ of the total emulsion volume. This figure represents the maximum volume ratio of uniform droplets packed in the most compact manner ${ }^{2}$. Generally, concentrated emulsions consist of continuous phase, which contains monomers mixed with suitable crosslinker and surfactant and droplet phase, which comprises a solution of initiator and electrolyte in distilled water. After the polymerization of the continuous phase and the removal of the droplet phase, polymer foams with open-celled structures were produced.

The polymer foams prepared via concentrated emulsion template method had been considered for many applications, such as filtration media, catalyst supports, tissue engineering scaffold and ion-exchange resins due to their unique properties $^{3,5}$. By far, the most widely investigated polymer foams prepared via concentrated emulsion template method were made of styrene and divinylbenzene as the continuous phase. Recently, more porous materials had been synthesized using other monomers, such as butyl acrylate, benzyl chloride and methacrylate ${ }^{2,4}$. However, it was difficult to prepare porous materials using more hydrophilic monomers, such as acrylonitrile, because the emulsion prepared using acrylonitrile as continuous phase was unstable and separate easily into two phases before polymerization without additional measures.

In order to improve the emulsion stability, silica nanoparticles were incorporated into the emulsion. This paper describes the preparation of porous polyacrylonitrile materials with the aid of silica nanoparticles via concentrated emulsion template methods. Acrylonitrile was chosen as the monomer since polyacrylonitrile foams are often used as precursors for porous carbon materials. Therefore, it is possible to design the pore sizes and porous structures of the porous carbon materials through the synthesis of porous polymer templates.

\section{EXPERIMENTAL}

A typical procedure was as follows: The droplet phase consisted of distilled water, calcium chloride, potassium persulfate and silica particles was added dropwise to the continuous phase containing acrylonitrile, divinylbenzene and sorbitan monooleate (Span80) as surfactant in a flask under stirring. Polymerization occurred at $60{ }^{\circ} \mathrm{C}$ in an oven for $24 \mathrm{~h}$. The resulting solid foams were purified in a Soxlet apparatus with alcohol to remove the remaining surfactant and monomers. Then the foams were dried in a vacuum oven at $80{ }^{\circ} \mathrm{C}$ for $48 \mathrm{~h}^{5,7}$. 
To investigate the morphology of the resulting samples, SEM images were performed on JSM-6610LV microscope (JEOL,Japan) operated at an accelerating voltage of $20 \mathrm{kV}$. $\mathrm{N}_{2}$ adsorption-desorption isotherms were measured using a Micromeritics analyzer system (Tristar-3000) at $77 \mathrm{~K}$.

\section{RESULTS AND DISCUSSION}

The stability of the concentrated emulsions was the main problem we had to face during preparation of porous polyacrylonitrile materials. As we know, a stable concentrated emulsion can only be produced when the monomers used as the continuous phase were sufficiently hydrophobic ${ }^{4,6}$. However, the monomer, acrylonitrile used in the experiment was not very hydrophobic. The emulsion produced using acrylonitrile as the continuous phase without additional measures was unstable, which lacks the ability to maintain its macroscopic homogeneity and will collapse before complete polymerization. For this reason, silica nanoparticles were introduced into the droplet phase to enhance the emulsion stability during preparation.

Fig. 1 showed the morphologies of polyacrylonitrile foams prepared with different silica nanoparticle concentrations in droplet phase. It was found from Fig. 1 that all the foams had a typical open-cell, highly interconnected porous structures and the silica particles accumulated on the surface of the foams without destroying the open porous structures. In the experiment, the incorporation of the silica nanoparticles had greatly enhanced the emulsion stability and foams could be easily prepared using concentrated emulsion template methods. Fig. 1(b) indicated that a suitable concentration of silica nanoparticles (about 4 wt. \%) in the droplet phase may well stabilize the concentrated emulsions and foams with uniform cells $(3-5 \mu \mathrm{m})$ were produced. It was found from Fig. 1(a) that if the concentration of silica particles was too low (2 wt. \%), coalescence of the droplet phase was serious and the emulsion became unstable, which resulted into the porous materials with larger $(2-9 \mu \mathrm{m})$ cells. However, it was noticed from Fig. 1(c) that at high concentration of the silica particles (7.5 wt. $\%)$, the viscosity of the concentrated emulsion became higher and it was difficult to generate a uniform, fine dispersion of the droplet phase. Therefore, the cells of the polymer foams prepared at high loading of silica nanoparticles became smaller (1-4 $\mu \mathrm{m})$ and more polydisperse. These observations indicated that the silica nanoparticles, which were absorbed on the emulsion interface, had acted as a cosurfactant preventing the coalescence of the droplets and enhanced the emulsion stability ${ }^{7,9}$.

The feature of the concentrated emulsion was that the volume ratio of droplet phase of the concentrated emulsion was higher than $74 \%$. When the volume ratio of droplet phase was sufficiently high, the droplets were no longer uniform in size and deformed into polyhedra. Fig. 2 showed the morphologies of the porous polyacrylonitrile materials prepared with different volume ratio of droplet phase.

It was found from Fig. 2 that raising the volume ratio of the droplet phase from 0.74 to 0.90 , led to an increase in cell diameter from 4 to $10 \mu \mathrm{m}$. It was known that the increase of the volume ratio of the droplet phase was accompanied by the decrease of the amount of surfactant and silica nanoparticles on per unit interface area. Therefore, the higher the volume ratio of the droplet phase, the more unstable the emulsion was. The poorer emulsion stability resulted in foams with larger cells. It was found from Fig. 2(c) that the cell diameters of
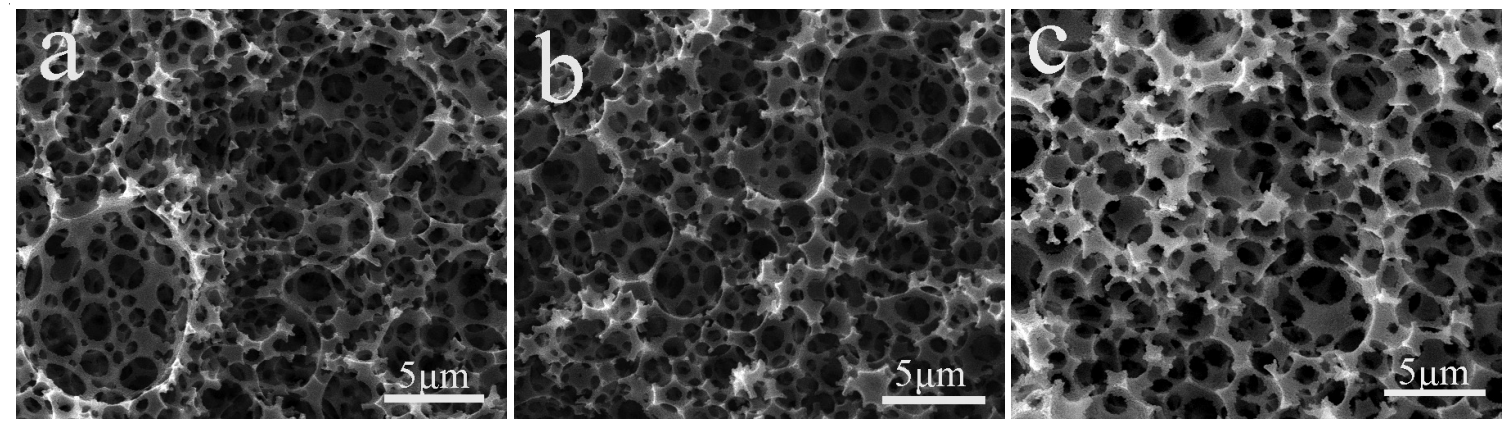

Fig. 1. Scanning electron micrographs of polyacrylonitrile foams based on different silica concentrations: (a) 2 wt. $\%$, (b) 4 wt. $\%$, (c) 7.5 wt. $\%$ (volume ratio of droplet phase: $80 \%$ )
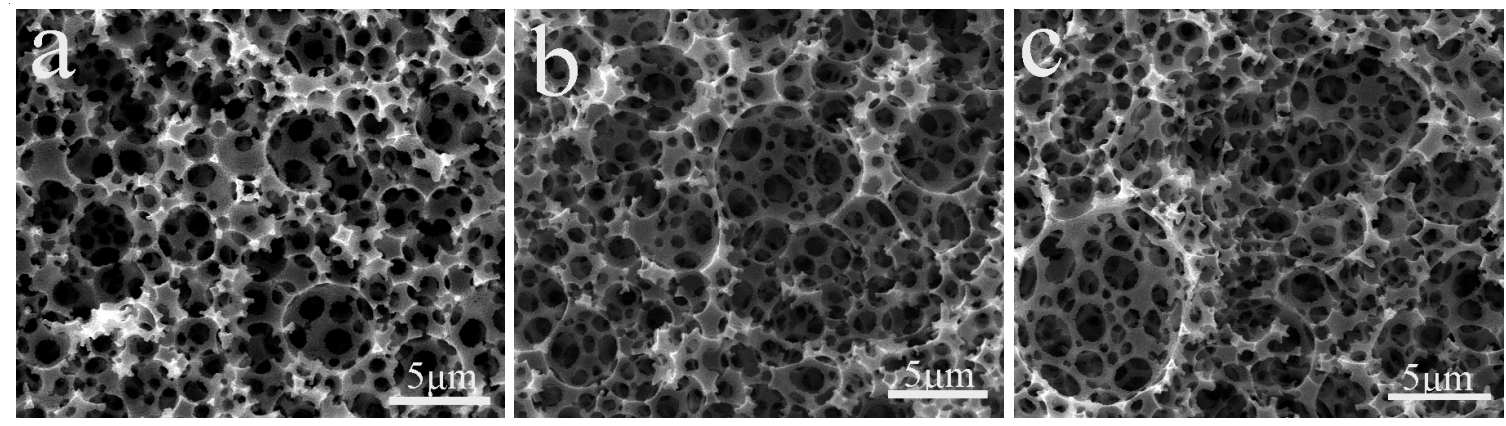

Fig. 2. Scanning electron micrographs of polyacrylonitrile foams based on different volume ratio of droplet phase: a 0.74 , b 0.82 , c 0.90 (silica concentration: 4 wt. \%) 

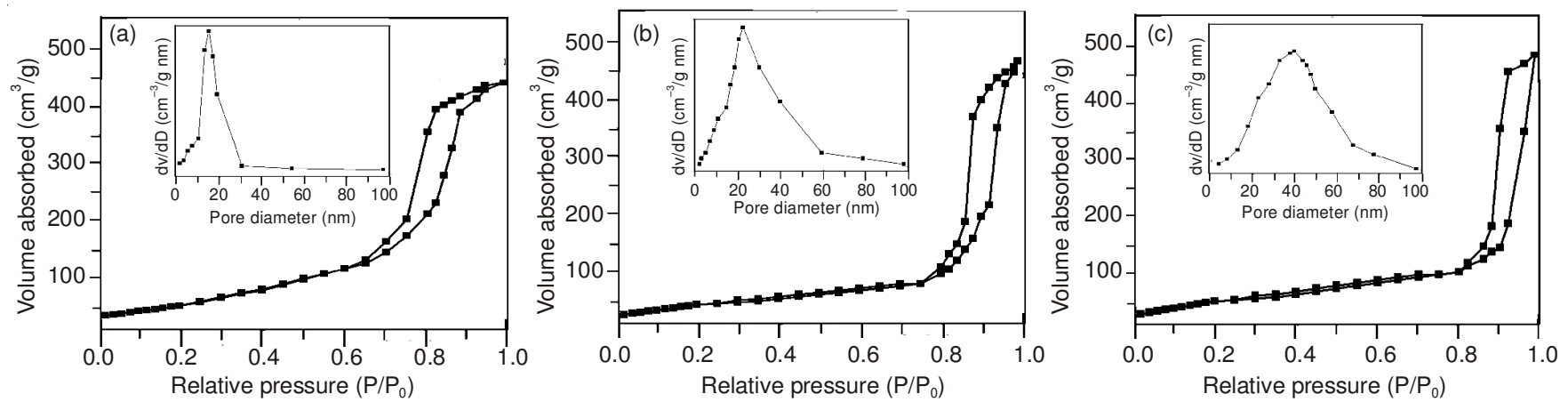

Fig. 3. Nitrogen adsorption-desorption isotherms and pore size distribution curves of porous polyacrylonitrile foams based on different volume ratio of droplet phase: (a) 0.74 , (b) 0.82 , (c) 0.90

polyacrylonitrile materials prepared at high volume ratio $(90 \%)$ of the droplet phase became larger $(8-10 \mu \mathrm{m})$ compared to Fig. 2(a, b) due to the serious coalescence of the droplets ${ }^{8,9}$.

Porous polyacrylonitrile materials have dual pore porosities: Large cells which are typical of this kind of materials and much smaller pores connecting adjacent cells of the foams. To investigate the porous structures of the polyacrylonitrile foams nitrogen adsorption-desorption isotherms were used $^{10,11}$. Fig. 3 showed the nitrogen adsorption-desorption isotherms and pore size distribution curves of polyacrylonitrile foams based on different volume ratio of droplet phase.

It was noticed form Fig. 3 that the out curves of the three samples exhibited the typical type-IV isotherms (IUPAC classification) indicating the presence of mesopores ${ }^{3,12,13}$. Hysteresis loops were observed at higher relative pressure $\left(\mathrm{p} / \mathrm{p}_{0}=0.82-0.99\right)$ from Fig. 3(b, c) suggesting larger average pore diameters and broad pore size distributions compared to Fig. 3(a). The inset showed that the average pore size increased from 19 to $48 \mathrm{~nm}$ based on different volume ratio of the droplet phase. This was attributed to the emulsion stability. Increasing the volume ratio of the droplet phase, the emulsion stability became poorer, which resulted into the foams with larger and more polydispersed pores ${ }^{14,15}$.

\section{Conclusion}

Porous polyacrylonitrile materials had been prepared via concentrated emulsion templates method. Stable concentrated emulsions were obtained with the aid of silica nanoparticles. We found that silica particles acted as a cosurfactant and tended to accumulated on the interface of the emulsion preventing the coalescence of the droplets, which efficiently improved the emulsion stability. The resulting foams posses typical interconnected network structure and the morphology of the foams was conveniently controlled by altering the emulsion composition.

\section{ACKNOWLEDGEMENTS}

The authors thank the Project of Shandong Province Higher Educational Science and Technology Program of China (Grant No. J14LA07) for their support.

\section{REFERENCES}

1. A. Barbetta and N.R. Cameron, Macromolecules, 37, 3202 (2004).

2. M.R. Moghbeli and M. Shahabi, Iranian Polym. J., 20, 343 (2011).

3. J.L. Wang, Z.J. Du, C.J. Li, H.Q. Li and C. Zhang, J. Appl. Polym. Sci., 111, 746 (2008).

4. Z. Abbasian and M.R. Moghbeli, J. Appl. Polym. Sci., 119, 3728 (2011).

5. A. Menner, K. Haibach, R. Powell and A. Bismarck, Polymer, 47, 7628 (2006).

6. J.R. Du, M.A. Hoisington, D.A. Langlois and B.C. Benicewicz, Polymer, 18, 4369 (1998).

7. Y.Y. Ma, P.N. Jia, X.C. Li, N. Liu and Y.L. Ma, J. Porous Mater., 19, 1047 (2012).

8. M. Akia, S.M. Alavi, M. Rezaei and Z.F. Yan, J. Porous Mater., 17, 85 (2010)

9. R. Tank and D.C. Gupta, J. Porous Mater., 16, 387 (2009).

10. N.R. Cameron and D.C. Sherrington, J. Mater. Chem., 7, 2209 (1997).

11. P. Krajnc, J.F. Brown and N.R. Cameron, Org. Lett., 4, 2497 (2002).

12. W. Busby, N.R. Cameron and A.B. Jahoda, Polym. Int., 51, 871 (2002).

13. M. Ottens, G. Leene, M. Beenackers, N. Cameron and D.C. Sherrington, Ind. Eng. Chem. Res., 39, 259 (2000).

14. A. Mercier, H. Deleuze and O. Mondain-Monval, Macromol. Chem. Phys., 202, 2672 (2001).

15. L. Moine, H. Deleuze and B. Maillard, Tetrahedron Lett., 44, 7813 (2003). 